\title{
Research on DOA Estimation of Multi-Component LFM Signals Based on the FRFT
}

\author{
Haitao QU ${ }^{1}$, Rihua WANG ${ }^{2}$, Wu QU ${ }^{3}$, Peng ZHAO ${ }^{4}$ \\ ${ }^{1}$ Beijing University of Posts and Telecommunications, Beijing, China \\ ${ }^{2}$ Communication University of China, Beijing, China \\ ${ }^{3} K$-Touch Corporation, Beijing, China \\ ${ }^{4}$ Beijing Research Institute of China Telecom Co., Ltd, Beijing, China \\ E-mail: quhaitao2007@gmail.com \\ Received April 10, 2009; revised May 20, 2009; accepted May 25, 2009
}

\begin{abstract}
A novel algorithm for the direction of arrival (DOA) estimation based on the fractional Fourier transform (FRFT) is proposed. Firstly, using the properties of FRFT and mask processing, Multi-component LFM signals are filtered and demodulated into a number of stationary single frequency signals. Then the one-dimensional (1-D) direction estimation of LFM signals can be achieved by combining with the traditional spectrum search method in the fractional Fourier (FRF) domain. As for the multi-component LFM signals, there is no cross-term interference, the mean square error (MSE) and Cramer-Rao bound (CRB) are also analyzed which perfects the method theoretically, simulation results are provided to show the validity of our method. The proposed algorithm is also extended to the uniform circular array (UCA), which realizes the two-dimensional (2-D) estimation. Using the characteristics of time-frequency rotation and demodulation of FRFT, the observed LFM signals are demodulated into a series of single frequency ones; secondly, operate the beam-space mapping to the single frequency signals in FRF domain, which UCA in array space is changed into the virtual uniform circular array (ULA) in mode space; finally, the DOA estimation can be realized by the traditional spectral estimation method. Compared with other method, the complex time-frequency cluster and the parameter matching computation are avoided; meanwhile enhances the estimation precision by a certain extent. The proposed algorithm can also be used in the multi-path and Doppler frequency shift complex channel, which expands its application scope. In a word, a demodulated DOA estimation algorithm is proposed and is applied to 1-D and 2-D angle estimation by dint of ULA and UCA respectively. The detailed theoretical analysis and adequate simulations are given to support our proposed algorithm, which enriches the theory of the FRFT.
\end{abstract}

Keywords: DOA Estimation, The Fractional Fourier Transform, UCA, ULA, LFM

\section{Introduction}

In various applications of array signal processing such as radar, sonar, communications, and seismology, there is a growing interest in estimating the DOA of LFM signals by dint of time-frequency analysis tools. G. Wang [1] proposed an iterative algorithm based on time-compensation, but the initial estimate is necessary. Using interpolation in the spatial time-frequency distribution matrices (STFD's) [2], Gershman [3] extended the signal subspace technique and estimated effectively DOA of
LFM signals, however Gershman's approach presences model biases in addition to time consuming. The above Wigner-Ville distribution (WVD) based methods consequentially suffer from the disturbance of cross-terms in the presence of multi-component signals.

Using a new time-frequency analysis tool-FRFT, direction estimation of LFM signals has been proposed in Reference [4]. However, only maximal energy concentration point is selected as estimate data, easily interfered by surroundings. In this paper, a new FRFT based algorithm is proposed. Firstly, Observed signals are separated into a number of single components by adding an adap- 
tive filter in the FRF domain. Secondly, the separated components are demodulated into stationary signals. Finally, the 1-D DOA of LFM signals can be estimated by the traditional spectrum search method. This algorithm digs two dimensional time and frequency information without the initial estimate, frequency focusing and parameter partnership. With the increasing of the Signal-to-Noise ratio (SNR), the MSE is quite closed to the CRB [5], for multi-component signals, cross-terms and non-linear optimize operation are also avoided.

For the UCA widely used in the third generation mobile communication system, the time-frequency characteristics of the FRFT are combined with the beamforming technology in FRF domain, an algorithm for the 2-D DOA estimation of the multi-component LFM signals is also proposed. Compared with other methods, the precision is enhanced by a certain extent. Simulation verifies the method to be effective in the multipath and Doppler frequency shift existed complex channels.

\section{Background Knowledge of FRFT}

\subsection{Definition and Properties of FRFT}

Recently the FRFT attracts more and more attention in the signal processing society, in 1980, Namias [6] firstly introduced the mathematical definition of the FRFT. Then Almeida [7] analyzed the relationship between the FRFT and the WVD, and interpreted it as a rotation operator in the time-frequency plane. This characteristic makes FRFT especially suitable for the processing of LFM signals [8-9].

As a generalization of the standard Fourier transform, the FRFT can be regarded as a counterclockwise rotation of the signal coordinates around the origin in the timefrequency plane. If the traditional Fourier transform of a signal can be considered as a $\pi / 2$ counterclockwise rotation from the time axis to the frequency axis, the FRFT can be accordingly considered as a counterclockwise rotation from the time axis to the $u$ axis with an angle $\alpha$, as illustrated by Figure 1 .

The FRFT of signal $x(t)$ is represented as

$$
X_{\alpha}(u)=F^{P}[x(t)]=\int_{-\infty}^{\infty} x(t) K_{\alpha}(t, u) d t
$$

where $p$ is called the order of the FRFT, $\alpha=p \pi / 2$, $F^{p}[\bullet]$ denotes the FRFT operator and $K_{\alpha}(t, u)$ is the kernel function of the FRFT

$$
K_{\alpha}(t, u)=\left\{\begin{array}{cc}
\sqrt{\frac{1-j \cot \alpha}{2 \pi}} \exp \left(j \frac{t^{2}+u^{2}}{2} \cot \alpha\right. \\
-j t u \csc \alpha), & \alpha \neq n \pi \\
\delta(t-u), & \alpha=2 n \pi \\
\delta(t+u), & \alpha=(2 n+1) \pi
\end{array}\right.
$$

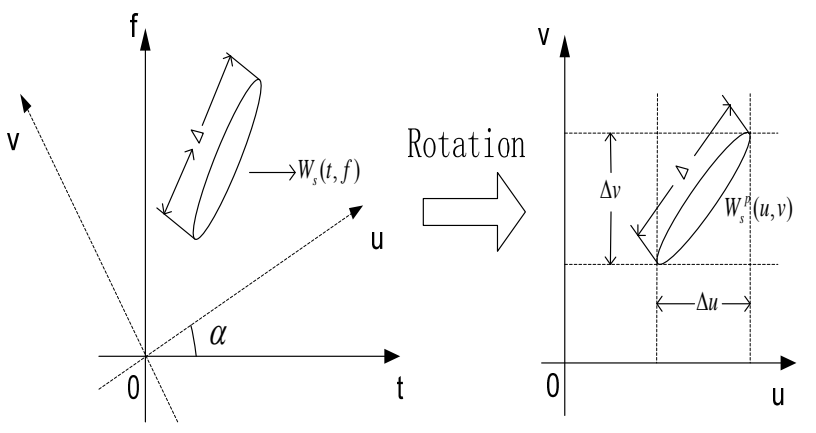

Figure 1. FRFT and WVD.

This has the following properties,

$$
\begin{gathered}
K_{-\alpha}(t, u)=K_{\alpha}^{*}(t, u) \\
\int_{-\infty}^{\infty} K_{\alpha}(t, u) K_{\alpha}^{*}\left(t, u^{\prime}\right) d t=\delta\left(u-u^{\prime}\right)
\end{gathered}
$$

Hence, the inverse FRFT is

$$
x(t)=F^{-P}[X(u)]=\int_{-\infty}^{\infty} X(u) K_{-\alpha}(t, u) d u
$$

Equation (5) indicates that signal $x(t)$ can be interpreted as decomposition to a basis formed by the orthonormal LFM functions in the $u$ domain, and the $u$ domain is usually called the fractional Fourier domain, in which the time and frequency domains are its special cases. The FRFT is a one-dimension linear transform and has the rotation-addition property. Essentially, the representation of a signal in the fractional domains contains the information in both time and frequency domains of the signal; Thus the FRFT is considered as a time-frequency analysis method and has close relationships with other time-frequency analysis tools.

In Reference [10], some important characteristics are expressed as

$$
\begin{gathered}
F^{p}\left[e^{j c t^{2} / 2}\right]=\sqrt{\frac{1+j \tan \alpha}{1+c \tan \alpha}} \exp \left(\frac{u^{2}}{2} \frac{c-\tan \alpha}{1+c \tan \alpha}\right) \\
F^{p}\left[x(t) e^{j v t}\right]=X_{p}(u-v \sin \alpha) \sqrt{b^{2}-4 a c} \\
\exp \left[-j\left(\frac{v^{2}}{2} \sin \alpha \cos \alpha+u v \cos \alpha\right)\right] \\
F^{p}[x(t-\tau)]=X_{p}(u-\tau \cos \alpha) \\
\exp \left[j\left(\tau^{2} \sin \alpha \cos \alpha / 2-u \tau \sin \alpha\right)\right]
\end{gathered}
$$

\subsection{Discrete FRFT Computation}

In engineering applications, the discrete FRFT (DFRFT) is usually required. According to the definition of the FRFT, it is obvious that the numerical computation of the DFRFT is much more complicated than that of DFT. So far, there have been several DFRFT algorithms with 


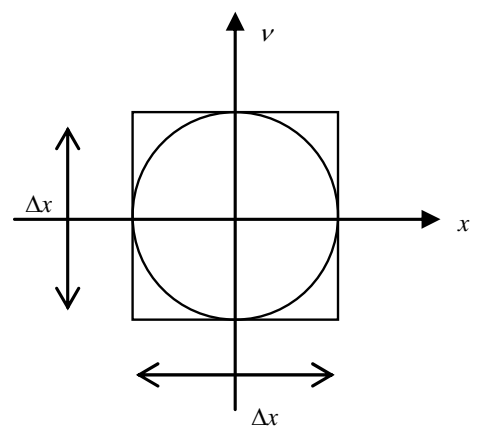

Figure 2. Normalized time-frequency support region.

different accuracies and different complexities. In this paper, we select the decomposition algorithm proposed in Reference [11]. This algorithm decomposes the computation of DFRFT to a convolution which can be computed by FFT, and the result is very close to the output of continuous FRFT. In this algorithm, the signal representation in time domain and frequency domain should be approximately constrained with an interval of $[-T / 2$, $T / 2]$ and a bandwidth of $[-F / 2, F / 2]$ respectively, viz. the time-bandwidth product of the signal is $N=T F$, and according to the uncertainty principle, $N>1$ constantly. If the sampling rate is selected as $T_{s}=T / \sqrt{N}$, the discrete representations of the signal in time domain and frequency domain will have the same length, which is called the dimensionless normalized process and the principle can be shown in Figure 2.

Therefore, Equation (1) can be expressed as

$$
X_{\alpha}(u)=A_{\alpha} e^{j \pi u^{2} \cot \alpha} \int_{-\infty}^{\infty} e^{j 2 \pi \csc \alpha u t} e^{j \pi t^{2} \cot \alpha} x(t) d t
$$

where

$$
A_{\alpha}=\sqrt{\frac{1-j \cot \alpha}{2 \pi}}
$$

For $0.5<|p|<1.5$, signal $e^{j \pi t^{2} \cot \alpha} x(t)$ has a bandwidth which is at most $2 F$ and can be represented using Shannon formula

$$
e^{j \pi t^{2} \cot \alpha} x(t)=\sum_{n=-N}^{N} e^{j \pi n^{2} \cot \alpha /(2 F)^{2}} x\left(\frac{n}{2 F}\right) \cdot \sin c\left(2 F\left(t-\frac{n}{2 F}\right)\right)
$$

Substituting Equation (10) into Equation (9) and exchanging the sequence of the integral and the summation, we have

$$
\begin{aligned}
& X_{\alpha}(u)=F^{P}[x(t)] \\
& =\frac{A_{\alpha}}{2 F} e^{j \pi u^{2} \cot \alpha} \sum_{n=-N}^{N} e^{-j 2 \pi u n \csc \alpha /(2 F)} e^{j \pi n^{2} \cot \alpha /(2 F)^{2}} \times\left(\frac{n}{2 F}\right)
\end{aligned}
$$

By quantizing the variable $u$ in the fractional Fourier domain, Equation (11) can be finally discredited as

$$
X_{\alpha}(m)=F^{P}\left[x\left(\frac{n}{2 F}\right)\right]=\frac{A_{\alpha}}{2 F} \sum_{n=-N}^{N} e^{j \pi\left(\gamma m^{2}-2 \beta m n+\gamma n^{2}\right) /(2 F)^{2}} x\left(\frac{n}{2 F}\right)
$$

where $X_{\alpha}(m)$ denotes the DFRFT of signal $x(t)$, $\gamma=-\cot \alpha, \beta=\csc \alpha$. This algorithm can be implemented by FFT, and has a computation complexity of $\mathrm{O}\left(N \log _{2} N\right)[11]$.

\subsection{Two Special FRF Domain}

WVD is an important non-stationary signal analysis tool, which has a very simple relationship with FRFT; viz. the WVD of FRFT is the coordinate rotation of the original signal' WVD, while the shape of WVD keeps unchanged in the rotation. Therefore, a lot of the WVD-based signal processing methods can be substituted by FRFT. The relationship of the two time-frequency analysis tools can draw a conclusion that "time width $(\Delta u)$ " and "frequency width $(\Delta v)$ " will change with the difference of the rotation angle. Considering two extreme cases, $\Delta u \rightarrow 0, \Delta v \rightarrow \Delta$ or $\Delta v \rightarrow 0, \Delta u \rightarrow \Delta$, from the above analysis, the former corresponds to the rotation angle $\alpha=-\cot ^{-1} \mu$, LFM signal becomes an impact function, which domain is called energy concentrated FRF one. The latter corresponds to the rotation angle $\alpha=-\cot ^{-1} \mu \pm \pi / 2$, LFM becomes a single frequency signal, which domain is called demodulated FRF one and is the base of the proposed algorithm in this paper. By dint of the time-frequency rotation property of FRFT, the detection, extraction and parameter estimation of LFM signals can be easily achieved.

\subsection{The FRFT of Gaussian White Noise}

Theorem 1: The FRFT of zero-mean Gaussian white noise is still Gaussian white noise.

Proof: let $n(t)$ subject to the $N\left(0, \sigma^{2}\right)$ distribution, and $N_{p}(u)$ is its FRFT, the mean is

$$
E\left\{N_{p}(u)\right\}=E\left\{F^{p}[n(t)]\right\}=F^{p}\{E[n(t)]\}=0
$$

Because the FRFT is the linear transform, does not change the distribution characteristics of Gaussian noise. Therefore, the noise is still a zero mean Gaussian noise.

As for the second-order statistical properties of noise, the correlation of the white noise $n(t)$ can be defined as:

$$
E\left\{n(t) n^{*}(t-\tau)\right\}=\sigma^{2} \delta(\tau)
$$


The correlation of $N_{p}(u)$ is defined as:

$$
\begin{aligned}
& E\left\{N_{p}(u) N_{p}^{*}(u-v)\right\} \\
& =E\left\{\int_{-\infty}^{+\infty} n(t) K_{p}(t, u) d t \int_{-\infty}^{+\infty} n^{*}(\lambda) K_{p}^{*}(\lambda, u-v) d \lambda\right\} \\
& =\int_{-\infty}^{+\infty} \int_{-\infty}^{+\infty} E\left\{n(t) n^{*}(\lambda)\right\} K_{p}(t, u) K_{p}^{*}(\lambda, u-v) d t d \lambda \\
& =\int_{-\infty}^{+\infty} \int_{-\infty}^{+\infty} \sigma^{2} \delta(t-\lambda) K_{p}(t, u) K_{p}^{*}(\lambda, u-v) d t d \lambda \\
& =\sigma^{2} \int_{-\infty}^{+\infty} K_{p}(\lambda, u) K_{p}^{*}(\lambda, u-v) d \lambda
\end{aligned}
$$

Submit Equation (2) to Equation (15), and obtain:

$$
\begin{aligned}
& E\left\{N_{p}(u) N_{p}^{*}(u-v)\right\} \\
& =\sigma^{2}\left|\sqrt{\frac{1-j \cot \alpha}{2 \pi}}\right|^{2} \mathrm{e}^{j 0.5\left(2 u v-v^{2}\right) \cot \alpha} \int_{-\infty}^{+\infty} \mathrm{e}^{-j \lambda v \csc \alpha} d \lambda \\
& =2 \pi \sigma^{2}\left|\sqrt{\frac{1-j \cot \alpha}{2 \pi}}\right|^{2}|\sin \alpha| \delta(v)
\end{aligned}
$$

Due to Equation (16), we can see that the FRFT does not change the time-domain white characteristics of noise, while noise energy does not be changed.

Assume the array noise is the zero-mean airspace one, viz. as for the array element $\mathrm{k}(k \neq l)$, the output noise is unrelated:

$$
E\left\{n_{k}(t) n_{l}^{*}(t)\right\}=E\left\{n_{k}(t)\right\} E\left\{n_{l}^{*}(t)\right\}=0
$$

The cross-correlation of the noise in FRF domain is

$$
\begin{aligned}
& E\left\{N_{k}^{p}(u)\left[N_{l}^{p}(u)\right]^{*}\right\} \\
& =\int_{-\infty}^{+\infty} \int_{-\infty}^{+\infty} E\left\{n_{k}(t) n_{l}^{*}(\lambda)\right\} K_{p}(t, u) K_{p}^{*}(\lambda, u) d t d \lambda \\
& =0
\end{aligned}
$$

The above equation shows, FRFT does not change the airspace white characteristics of noise. Therefore, we can draw a conclusion that FRFT does not change the statistical properties of Gaussian white noise, the theorem certification has completed.

Inference: as for the $M$ antenna array element, if the array output noise is zero mean and variance $\sigma^{2}$, the noise covariance matrix in FRF domain is:

$$
R_{N}^{p}=E\left\{N_{p}(u) N_{p}^{*}(u)\right\}=\sigma^{2} I_{M}
$$

\section{1-D DOA Estimation Algorithm}

\subsection{ULA Array Model}

Let a ULA of $M$ sensors receive LFM sources from the $D$ unknown directions $\left\{\theta_{1}, \theta_{2}, \cdots, \theta_{D}\right\}$, as illustrated by

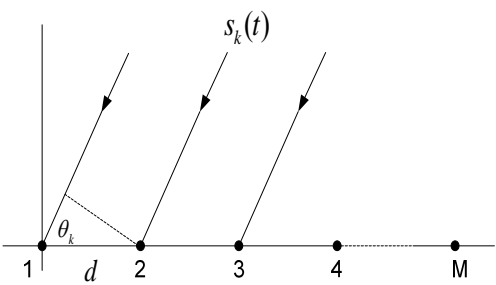

Figure 3. ULA and array model.

Figure 3. The observed signal at the output of the $i$ th sensor can be described as

where,

$$
\begin{gathered}
x_{i}(t)=\sum_{k=1}^{D} s_{k}\left[t-\tau_{i k}\right]+n_{i}(t) \\
i=1,2, \cdots, M-1 \quad k=1,2, \cdots, D
\end{gathered}
$$

$$
\begin{aligned}
s_{k}(t) & =\exp \left[j\left(\omega_{k} t+\mu_{k} t^{2} / 2\right)\right] \\
\tau_{i k} & =(i-1) d \cos \theta_{k} / c
\end{aligned}
$$

$\omega_{k}, \mu_{k}$ are initial frequency and FM rate, $s_{k}(t)$ is the $k$ th source in reference sensor $x_{1} . n_{i}(t)$ is the additive white Gauss noise with variance $\sigma^{2}$, which is assumed to be statistically independent with signal sources. $\tau_{i k}$ is the $k$ th's path delay, $c$ is light velocity and $d$ is sensor spacing.

From (20) and (21), we get the direction matrix is time-variant; however the traditional estimation method is merely suitable for time-invariant signal model. Therefore, the traditional method cannot be used to the direction finding of LFM signals directly.

\subsection{1-D Estimation Algorithm Description}

In this section, the main work is how to make the direction matrix time-invariant. The FRFT is actually a "Rotation" of signal in time-frequency plane. An LFM signal can be turned into an impulse in a proper fractional domain, for the ULA model, signal $s_{k}(t)$ will present an impulse while the rotation angle $\alpha_{k^{\prime}}=\pi-\cot \mu_{k}$. There will be the energy concentration, consequently a distinct peak will appear in that FRF domain, whereas the noise energy is distributed much more symmetrically in the entire time-frequency plane and will not be concentrated in any FRF domain [12].

Using (20) and (21), we get that path delay can not change the FM rates, so the impulse corresponding rotation angles of signal $s_{k}(t)$ are same in every sensor. Then Equation (20) is rotated with angle $\alpha_{k^{\prime}}$ by the FRFT from two sides:

$$
W_{i}^{\alpha_{k^{\prime}}}\left(u^{\prime}\right)=Y_{i k^{k^{\prime}}}^{\alpha^{\prime}}\left(u^{\prime}\right)+\sum_{l \neq k}^{D} Y_{i l}^{\alpha_{k^{\prime}}}\left(u^{\prime}\right)+V_{i}^{\alpha_{k^{\prime}}}\left(u^{\prime}\right)
$$


where, $Y_{i k^{k^{\prime}}}^{\alpha^{\prime}}\left(u^{\prime}\right)$ presents an impulse, $\sum_{l \neq k}^{D} Y_{i l}^{\alpha_{k^{\prime}}}\left(u^{\prime}\right)$ and $V_{i}^{\alpha_{k^{\prime}}}\left(u^{\prime}\right)$ are approximately considered as LFM signal and the white Gauss noise respectively.

Therefore, a mask operation is applied to (23) according to the peak position $m_{i k}$, which is a narrowband filter with central frequency $m_{i k}$, and with a properly selected bandwidth $2 L$, most energy of the signal $Y_{i k^{\prime}}^{\alpha_{k^{\prime}}}\left(u^{\prime}\right)$ will be removed. This procedure can be regarded as an open loop adaptive time-varying filter whose central frequency varies linearly following the peak position $m_{i k}$.

Signal $Y_{i k^{\prime}}^{\alpha_{k^{\prime}}}\left(u^{\prime}\right)$ is performed the FFT (viz. FRFT of $p=1)$. According to the rotation-addition property [10], the two procedures above are equivalence to one time rotation with angle $\alpha_{k}$ viz.

$$
\begin{aligned}
\alpha_{k} & =3 \pi / 2-\cot \mu_{k} ; \\
\mu_{k} & =\tan \alpha_{k}
\end{aligned}
$$

Using (6), (7) and (8), signal $s_{k}(t)$ is rotated with angle $\alpha_{k}$ by the FRFT can be expressed as

$$
\begin{aligned}
& S_{k}^{\alpha_{k}}(u)= \\
& \quad \sqrt{\frac{1+j \tan \alpha_{k}}{1+\mu_{k} \tan \alpha_{k}} \exp \left[\frac{\left(u-\omega_{k} \sin \alpha_{k}\right)^{2}}{2} \frac{\mu_{k}-\tan \alpha_{k}}{1+\mu_{k} \tan \alpha_{k}}\right]} \\
& \exp \left[-j\left(\omega_{k}^{2} \sin \alpha_{k} \cos \alpha_{k} / 2+u \omega_{k} \cos \alpha_{k}\right)\right] \\
& =\sqrt{\frac{1+j \tan \alpha_{k}}{1+\mu_{k} \tan \alpha_{k}}} \exp \left[-j\left(\omega_{k}^{2} \sin \alpha_{k} \cos \alpha_{k} / 2\right)\right] \\
& \quad \exp \left(-j u \omega_{k} \cos \alpha_{k}\right)=B \exp \left(-j u \omega_{k} \cos \alpha_{k}\right)
\end{aligned}
$$

where,

$$
B=\sqrt{\frac{1+j \tan \alpha_{k}}{1+\mu_{k} \tan \alpha_{k}}} \exp \left[-j\left(\omega_{k}^{2} \sin \alpha_{k} \cos \alpha_{k} / 2\right)\right]
$$

From (25) and (26), it can be seen that LFM signal $s_{k}(t)$ has been transformed into the single frequency signal $S_{k}^{\alpha_{k}}(u)$ in the FRF domain.

Similarly, the FRFT of path delayed signal $s_{k}(t-\tau)$ with rotation angle $\alpha_{k}$ can be expressed as

$$
\begin{aligned}
& F^{\alpha_{k}}\left[s_{k}(t-\tau)\right]=B \exp \left(j \tau^{2} \sin \alpha_{k} \cos \alpha_{k} / 2\right) \\
& \quad \exp \left(j \tau \omega_{k} \cos ^{2} \alpha_{k}\right) \exp \left[-j u\left(\omega_{k} \cos \alpha_{k}+\tau \sin \alpha_{k}\right)\right]
\end{aligned}
$$

In practice, $\tau$ is too small viz.

$$
\tau \sin \alpha_{k} \square \omega_{k} \cos \alpha_{k}
$$

$$
\exp \left(j \tau^{2} \sin \alpha_{k} \cos \alpha_{k} / 2\right) \approx 0
$$

Substituting (28) into (27), we get

$$
\begin{aligned}
F^{\alpha_{k}}[ & \left.S_{k}(t-\tau)\right] \\
& \approx B \exp \left(j \tau \omega_{k} \cos ^{2} \alpha_{k}\right) \exp \left(-j u \omega_{k} \cos \alpha_{k}\right) \\
& =\exp \left(j \tau \omega_{k} \cos ^{2} \alpha_{k}\right) S_{k}^{\alpha_{k}}(u)
\end{aligned}
$$

From the above analysis, Using (25) and (29), the observed signals described by (20) are performed the FRFT with rotation angle $\alpha_{k}$ from two sides

$$
\begin{gathered}
X_{i k}^{\alpha_{k}}(u)=S_{i k}^{\alpha_{k}}(u)+N_{i k}^{\alpha_{k}}(u) \\
i=1,2, \cdots, M-1
\end{gathered}
$$

Equation (30) can be compactly represented by matrix form as follows

$$
\begin{gathered}
X_{k}^{\alpha_{k}}(u)=A_{k}^{\alpha_{k}} S_{k}^{\alpha_{k}}(u)+N_{k}^{\alpha_{k}}(u) \\
A_{k}^{\alpha_{k}}=\left[a_{1 k}, \cdots, a_{i k}, \cdots, a_{M k}\right]^{T}
\end{gathered}
$$

where, $T$ denotes the transpose of matrix.

$$
\begin{gathered}
a_{i k}=\exp \left(j \tau_{i k} \omega_{k} \cos ^{2} \alpha_{k}\right) \\
=\exp \left(j \frac{2 \pi}{\lambda} \cos ^{2} \alpha_{k}(i-1) d \cos \theta_{k}\right) \\
X_{k}^{\alpha_{k}}(u)=\left[X_{1 k}^{\alpha_{k}}(u), X_{2 k}^{\alpha_{k}}(u), \cdots, X_{M k}^{\alpha_{k}}(u)\right] \\
N_{k}^{\alpha_{k}}(u)=\left[N_{1 k}^{\alpha_{k}}(u), N_{2 k}^{\alpha_{k}}(u), \cdots, N_{M k}^{\alpha_{k}}(u)\right]
\end{gathered}
$$

From (32) and (33), the direction matrix $A_{k}^{\alpha_{k}}$ is only relative to the direction information $\theta_{k}$, so the observed signal model has been time-variant in the FRF domain.

In the FRF domain, the covariance matrix of the observed signal can be defined as

$$
R_{X X}^{\alpha_{k}}=E\left[X_{k}^{\alpha_{k}}(u) X_{k}^{\alpha_{k} H}(u)\right]=A_{k}^{\alpha_{k}} R_{S S}^{\alpha_{k}} A_{k}^{\alpha_{k} H}+\sigma^{2} I
$$

where, $H$ denotes the conjugate transpose of matrix. $R_{S S}^{\alpha_{k}}$ is the auto-correlation matrix of signal sources. The composite covariance matrix (35) has the same structure as the covariance matrix arising in the case of stationary signals. Therefore, the DOA can be estimated by performing eigendecomposition to $R_{X X}^{\alpha_{k}}$. Using the signal subspace $S_{N}^{\alpha_{k}}$ and the noise subspace $E_{N}^{\alpha_{k}}$, the space spectrum function of the $k$ th source in the FRF domain can be given by [13]

$$
P\left(\theta_{k}\right)=1 /\left(A_{k}^{\alpha_{k} H} E_{N}^{\alpha_{k}} E_{N}^{\alpha_{k} H} A_{k}^{\alpha_{k}}\right)
$$

$P\left(\theta_{k}\right)$ is performed an 1-D search and $\alpha_{k}$ can be obtain by the maximal peak rotation angle. Similarly, all the Direction of LFM signals can be estimated in turn. This 
algorithm is considered as FRFT based demodulation method.

To summarize, the proposed algorithm can be formulated as follows:

1) The observed signals at all sensors are rotated with a continuously variable angle $\alpha$ by the FRFT; perform a 2-D peak search in the $(\alpha, m)$ plan to obtain the maximal peak position $m_{i k}$ and corresponding rotation angle $\alpha_{k^{\prime}}$ respectively.

2) Mask operations are applied according to $m_{i k}$ at every sensor, then the filtered $2 L$ points are performed the FFT to obtain stationary signals consequently.

3) Get the covariance matrix of the stationary signals and perform eigendecomposition in the FRF domain, construct the spectrum function $P\left(\theta_{k}\right)$ according to (36).

4) Perform 1-D peak search to $P\left(\theta_{k}\right)$ and obtain the DOA of the $k$ th LFM signal.

5) For multi-component LFM signals, all the direction can be estimated by repeating the above procedures.

\section{2-D DOA Estimation Algorithm Using UCA}

\subsection{Introduction}

UCA has many advantages which the linear array cannot match. E.g. UCA can be implemented with all-directionfunding; its precision measurement does not change with the azimuth significantly and is fit for the system correcting. UCA is the main receiving antenna of base station system in the third generation mobile communication system. Thus, the UCA based DOA estimation has been a research hotspot in array signal processing. Mathwes [14] proposed an UCA-RB-MUSIC method, which can be only suitable for the stationary signals; however, the actually existed signals are non-stationary ones which are represented by LFM. Tao ran [4] proposed an algorithm of LFM signal DOA estimation. However, the method does not apply to the UCA.

Due to the above analysis, we propose a novel DOA estimation algorithm based on FRFT using UCA, as for the multi-component LFM signals, using the characteristics of time-frequency rotation and demodulation of FRFT. Firstly, the observed signals are demodulated into a series of single frequency ones; secondly, operate the beam-space mapping to the single frequency signals in FRF domain, which UCA in array space is changed into the virtual ULA in mode space; finally, the DOA estimation can be realized by the traditional spectral estimation method. The proposed algorithm mines the time, frequency and spatial information maximally; compared with other method, the complex time-frequency cluster and the parameter matching computation are avoided; meanwhile enhance the precision [15]. As for the multi-component LFM signals, there is no cross-term interference, the proposed algorithm is also applicable for the multi-path and Doppler frequency shift channels.

\subsection{UCA Array Model}

Assuming $D$ independent LFM signals and the pitch and azimuth angle is $\left\{\left(\theta_{1}, \phi_{1}\right),\left(\theta_{2}, \phi_{2}\right), \cdots,\left(\theta_{D}, \phi_{D}\right)\right\}$ respectively, the array element number of UCA is $N$ and radius is $r$, the center is the reference point of receiving antenna, as shown in Figure 4. Then the output of the $i$ th sensor is:

$$
\begin{array}{r}
x_{i}(t)=\sum_{k=1}^{D} s_{k}\left[t-\tau_{i k}\right]+n_{i}(t) \\
i=1,2, \cdots, N \quad k=1,2, \cdots, D
\end{array}
$$

where,

$$
\begin{aligned}
S_{k}(t) & =\exp \left[j\left(\omega_{k} t+\mu_{k} t^{2} / 2\right)\right] \\
\tau_{i k} & =r \sin \theta_{k} \cos \left(\phi_{k}-\varepsilon_{i}\right) / c \\
\varepsilon_{i} & =2 \pi(i-1) / N
\end{aligned}
$$

$s_{k}(t)$ is the $k$ th LFM source, and $\omega_{k}$ and $\mu_{k}$ are the initial frequency and FM rate respectively, $\tau_{i k}$ is the path delay and $c$ is the light velocity. $n_{i}(t)$ is the additive white Gaussian noise with zero mean and variance $\sigma^{2}$, which is independent with signals.

From the Equations (37) and (38), the direction matrix of observed signals is time-varying in UCA, while the traditional DOA estimation algorithm is only suitable for the time-invariant model, which cannot be used to deal with LFM signal directly.

\subsection{2-D Estimation Algorithm Description}

From Equations (26) and (28), operate the FRFT to Equation (37) with the rotation angle $\alpha_{k}$ from two sides:

$$
X_{i k}^{\alpha_{k}}(u)=S_{i k}^{\alpha_{k}}(u)+N_{i k}^{\alpha_{k}}(u)
$$

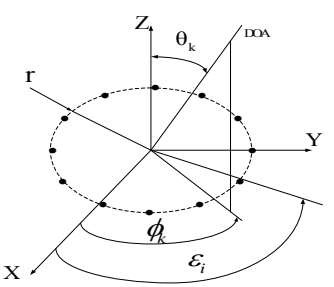

Figure 4. Uniform circular array. 
The matrix form of Equation (41) is:

$$
\begin{gathered}
X^{\alpha_{k}}(u)=A^{\alpha_{k}} S_{k}^{\alpha_{k}}(u)+N^{\alpha_{k}}(u) \\
A^{\alpha_{k}}=\left[a_{1 k}, \cdots, a_{i k}, \cdots, a_{N k}\right]^{T}
\end{gathered}
$$

where, $T$ donates the transpose of matrix.

$$
\begin{aligned}
& a_{i k}=\exp \left(j \tau_{i k} \omega_{k} \cos ^{2} \alpha_{k}\right) \\
& =\exp \left(j 2 \pi r \sin \theta_{k} \cos \left(\phi_{k}-\varepsilon_{i}\right) \cos ^{2} \alpha_{k} / \lambda\right)
\end{aligned}
$$

From Equations (43) and (44), in appropriate FRF domain, direction matrix $A^{\alpha_{k}}$ is only related to angle information $\phi, \theta$, viz. the observed signals have been transformed into unvaried smooth signal model. Therefore, the mode excitation method can be used to estimate the DOA of LFM signals.

The spatial beam former $F_{r}^{H}$ in FRF domain is defined as

$$
F_{r}^{H}=Q^{H} C e R^{H}
$$

where, $H$ denotes the conjugated transpose of matrix.

$$
\begin{gathered}
C e=\operatorname{diag}\left\{j^{-M}, \cdots, j^{-1}, j^{0}, j^{-1}, \cdots, j^{-M}\right\} \\
R^{H}=\sqrt{N}\left(Q_{-M}, \cdots, Q_{o}, \cdots, Q_{M}\right)^{H}
\end{gathered}
$$

Select the central Hilbert matrix,

$$
\begin{gathered}
Q=\frac{1}{\sqrt{M^{\prime}}}\left[v\left(\beta_{-M}\right), \cdots, v\left(\beta_{0}\right), \cdots, v\left(\beta_{M}\right)\right] \\
v(\psi)=\left[e^{-j M \psi}, \cdots, e^{-j \psi}, e^{j 0}, e^{j \psi}, \cdots, e^{j M \psi}\right] \\
\beta_{t}=2 \pi / M^{\prime} \quad t \in[-M, M]
\end{gathered}
$$

where, the largest model number $M \approx k r, M^{\prime}=2 M+1$. Wave number $k=2 \pi / \lambda, \quad \lambda$ is the initial frequency corresponding center wavelength of LFM signals. $F_{r}^{H}$ can change the UCA in the array space into the virtual ULA in the mode space, and finally, the DOA estimation can be achieved by the eigendecomposition based search method.

Summarize the above and the main steps are as follows:

1) The observed signals are continuously operated by FRFT; perform a 2-D peak search in the $(\alpha, m)$ plan to obtain the maximal peak position $m_{i k}$ and corresponding rotation angle $\alpha_{k^{\prime}}$ of the $k$ th LFM signal respectively.

2) Select $2 L$ points whose center is $m_{i k}$ and calculate the FFT (FRFT with $p=1$ ), obtain the $k$ th single frequency signal $X_{i k}^{\alpha_{k}}(u)$.

3) Let $X_{i k}^{\alpha_{k}}(u)$ pass the beam switch $F_{r}^{H}$, viz.

$$
\begin{aligned}
Y_{i k}^{\alpha_{k}}(u) & =F_{r}^{H} X_{i k}^{\alpha_{k}}(u) \\
= & F_{r}^{H} A^{\alpha_{k}} S_{k}^{\alpha_{k}}(u)+F_{r}^{H} N^{\alpha_{k}}(u),
\end{aligned}
$$

And calculate its covariance matrix

$R_{Y}=E\left[Y_{i k}^{\alpha_{k}}(u) Y_{i k}^{\alpha_{k}}(u)^{H}\right]$.

4) Define $R=\operatorname{Re}\left(R_{Y}\right)$, perform eigendecomposition to $R$ and obtain the signal subspace $S$ and noise subspace $G$. Construct:

$$
P\left(\theta_{k}, \phi_{k}\right)=\frac{1}{a_{i k}^{T}\left(\theta_{k}, \phi_{k}\right) G G^{T} a_{i k}\left(\theta_{k}, \phi_{k}\right)},
$$

where, $a_{i k}\left(\theta_{k}, \phi_{k}\right)=F_{r}^{H} a\left(\theta_{k}, \phi_{k}\right)$, perform 2-D

spectrum search and obtain $\theta_{k}$ and $\phi_{k}$.

5) As for the multi-component LFM signal, repeat the above process and obtain all the DOA of signals respectively.

\section{Performance Analysis and Simulation}

\subsection{FRFT Property Simulation}

\subsubsection{Simulation of FRFT and WVD}

As we all know, WVD is also one of the most important and most widely used time-frequency analysis tool, which is bound to FRFT with the existence of close ties. The derivation process is relatively complex; however, there is a very simple relationship between FRFT and WVD, that is, FRFT of WVD is the coordinate's rotation form of WVD of original signal [10].

In order to validate the relationship between the FRFT and WVD, experiments of compute simulations are given. We assume a wideband LFM signal $s(t)$ with a length of 1024, which is modeled as: initial frequency and FM rates are $\omega=9 \mathrm{MHz}, \mu=-0.7 \mathrm{MHz} / \mu \mathrm{s}$, sample frequency is $f_{s}=50 \mathrm{MHz}$. The WVD of $s(t)$ is shown in Figure $5(\mathrm{a}), s(t)$ is performed the FRFT by the rotation angle $0.15 \pi$ and get the transformed signal $S_{0.15 \pi}(u)$. The WVD of the transformed signal $S_{0.15 \pi}(u)$ is shown in Figure 5(b). Compared the two figures, it can be found that the WVD of $S_{0.15 \pi}(u)$ is just the rotation of the WVD of $s(t)$ by angle $0.15 \pi$, meanwhile the figure shape is invariable. So the FRFT is testified a kind of rotation arithmetic operators in the time-frequency plane.

\subsubsection{Two Special FRF Domain Simulation}

$s(t)=\exp \left[j\left(\omega t+\mu t^{2} / 2\right)\right]$, signal model is: $\omega_{1}=9 \mathrm{MHz}$, $\mu_{1}=-1400000 \mathrm{MHz} / \mathrm{s}$. Sampling rate $f_{s}=50 \mathrm{MHz}$, the number of snapshots is 1024. Perform continuous FRFT to signal and operate spectrum peak search, in the appropriate FRF domain, $s(t)$ shows the property of energy 


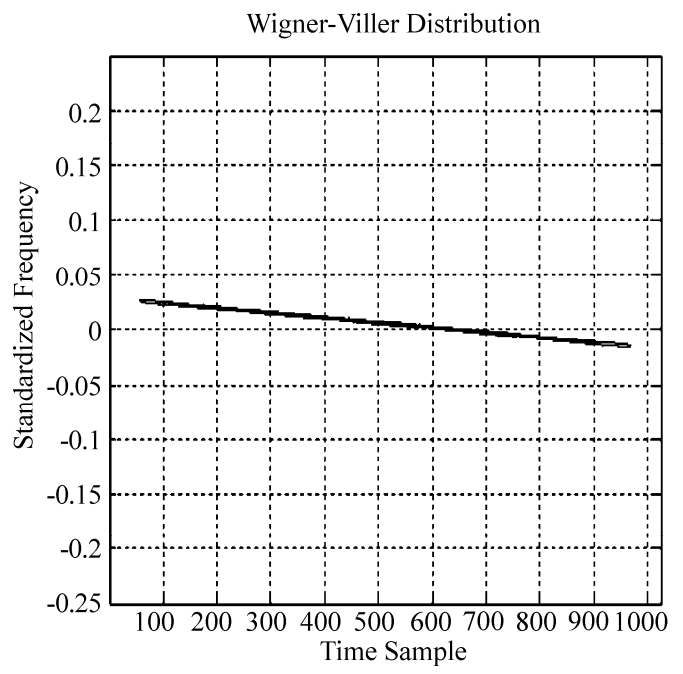

(a)

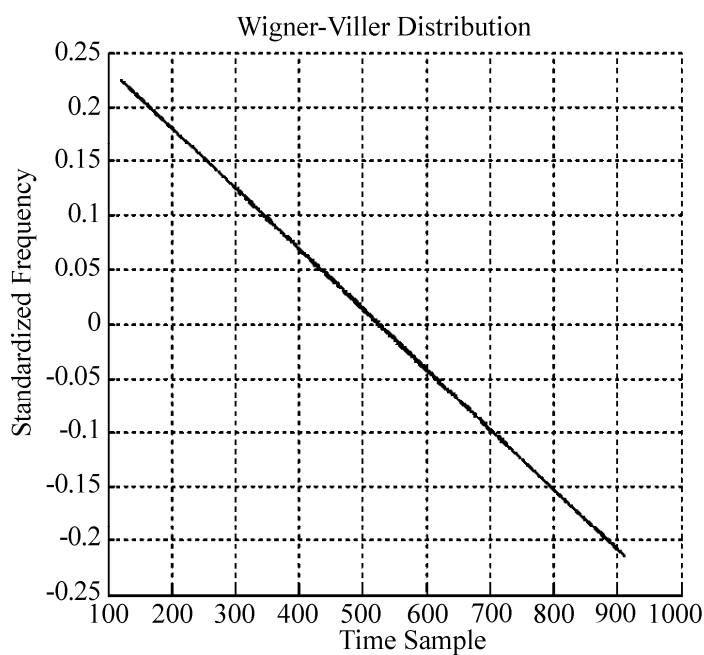

(b)

Figure 5. (a) The WVD of $s(t)$, (b) The WVD of $S_{0.15 \pi}(u)$.

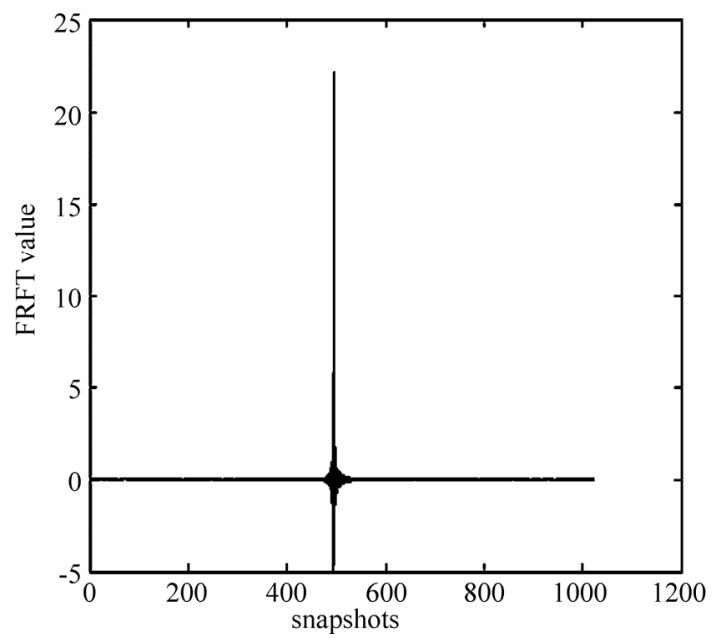

(a)

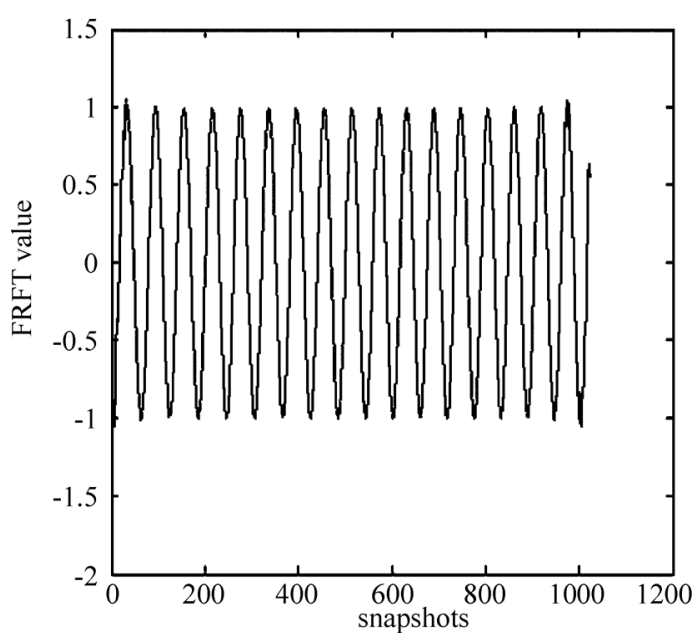

(b)

Figure 6. (a) Energy concentration property of FRFT, (b) Demodulated property of FRFT.

concentration, as shown in Figure 6(a). The signal continues to be rotated $\pi / 2$ in FRF domain, viz. in the demodulated FRF domain, $s(t)$ shows the demodulated property, as shown in Figure 6(b).

\subsubsection{Gaussian White Noise Simulation}

Assume the complex Gaussian white noise is: $w(n)=\operatorname{randn}(1,1024)+\operatorname{jrandn}(1,1024)$ and perform continuous FRFT to it, the energy distribution of $w(n)$ in different FRF domain is shown in Figure 7. We can see that the Gaussian white noise does not show energy concentration property in any FRF domain and can still be regarded as white noise. Thus theorem 1 is verified.

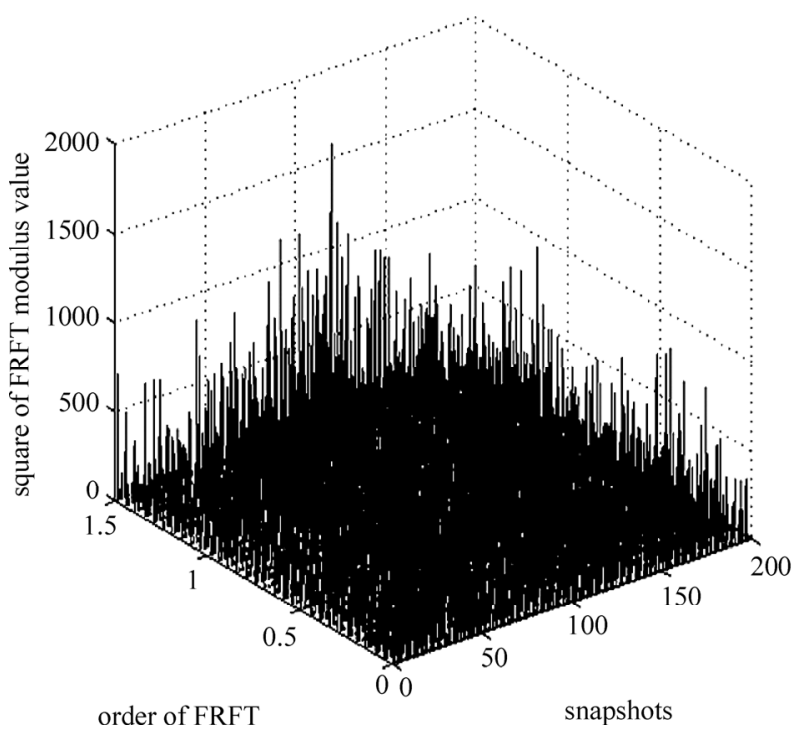

Figure 7. Energy distribution in different FRF domain. 


\subsection{1-D DOA Estimation Simulation}

\subsubsection{MSE and CRB Analysis}

The FRFT is a 1-D linear transform [10]. In the FRF domain $N_{k}^{\alpha_{k}}(u)$ is approximately considered as the additive Gauss white noise. Therefore, the probability density function of signal $X_{k}^{\alpha_{k}}(u)$ represents normal school and the corresponding likelihood function can be expressed as

$$
\begin{aligned}
& L\left[X_{k}^{\alpha_{k}}\right]=\frac{1}{(2 \pi)^{M}\left(\sigma^{2} / 2\right)^{M}} \\
& \exp \left\{-\frac{1}{\sigma^{2}}\left[X_{k}^{\alpha_{k}}-A_{k}^{\alpha_{k}} S_{k}^{\alpha_{k}}\right]^{H}\left[X_{k}^{\alpha_{k}}-A_{k}^{\alpha_{k}} S_{k}^{\alpha_{k}}\right]\right\}
\end{aligned}
$$

Using Reference [5], the CRB of the proposed method in the FRF domain can be represented as

$$
\begin{aligned}
& \operatorname{CRB}^{-1}\left(\theta_{k}\right) \\
& =\frac{2}{\sigma^{2}} \operatorname{Re}\left\{\left(S_{k}^{\alpha_{k}}\right)^{H} d_{k}^{H}(w)\left[I-A_{k}^{\alpha_{k}}\left(A_{k}^{\alpha_{k} H} A_{k}^{\alpha_{k}}\right)^{-1} A_{k}^{\alpha_{k} H}\right] d_{k}(w) S_{k}^{\alpha_{k}}\right.
\end{aligned}
$$

where, $\sigma^{2}$ is the noise variance and $I$ is unit matrix, $d_{k}(w)=d A_{k}^{\alpha_{k}} / d w$.

Similarly, the MSE of the proposed algorithm in the FRF domain can be represented as

$$
\begin{gathered}
V A R_{M U}^{-1}\left(\theta_{k}\right)=\frac{2}{\sigma^{2}}\left[d_{k}^{H}(w)\left[I-A_{k}^{\alpha_{k}}\left(A_{k}^{\alpha_{k} H} A_{k}^{\alpha_{k}}\right)^{-1} A_{k}^{\alpha_{k} H}\right]\right. \\
\left.d_{k}(w)\right] /\left\{\left[R_{X X}^{\alpha_{k}-1}\right]_{11}+\sigma^{2}\left[R_{X X}^{\alpha_{k}-1}\left(A_{k}^{\alpha_{k} H} A_{k}^{\alpha_{k}}\right)^{-1} R_{X X}^{\alpha_{k}-1}\right]_{11}\right\}
\end{gathered}
$$

where, $R_{X X}^{\alpha_{k}}$ is covariance matrix of the observed signals, []$_{11}$ denotes the first row and first line element of matrix.

From (52) and (53), it can be obtain that the MSE of the proposed method will be more and more closed to the $\mathrm{CRB}$ with the increasing of the sensor number and the SNR.

\subsubsection{MSE and CRB Simulation}

In order to validate the proposed method, experiments of compute simulations are given. We assume the ULA of $M=6$ impinging from $D=2$ far field wideband LFM signals with a length of 1024 , which is modeled as: initial frequency and FM rates are $\omega_{1}=200 \pi \mathrm{Hz}$,

$$
\mu_{1}=-900 \pi \mathrm{Hz} / \mathrm{s} ; \omega_{2}=200 \pi \mathrm{Hz}, \mu_{2}=300 \pi \mathrm{Hz} / \mathrm{s} \text {, }
$$
angles of arrival are $\theta_{1}=30^{\circ}$ and $\theta_{2}=70^{\circ}$ respectively. Sample frequency is $f_{s}=900 \mathrm{~Hz}$, the mask snapshots are $2 L=300$ in the FRF domain. The input SNR varies from $15 \mathrm{~dB}$ to $29 \mathrm{~dB}$ with an interval $2 \mathrm{~dB}$, at each level of the SNR, we run 100 Monte-Carlo experiments, the MSE of the proposed method and original method are

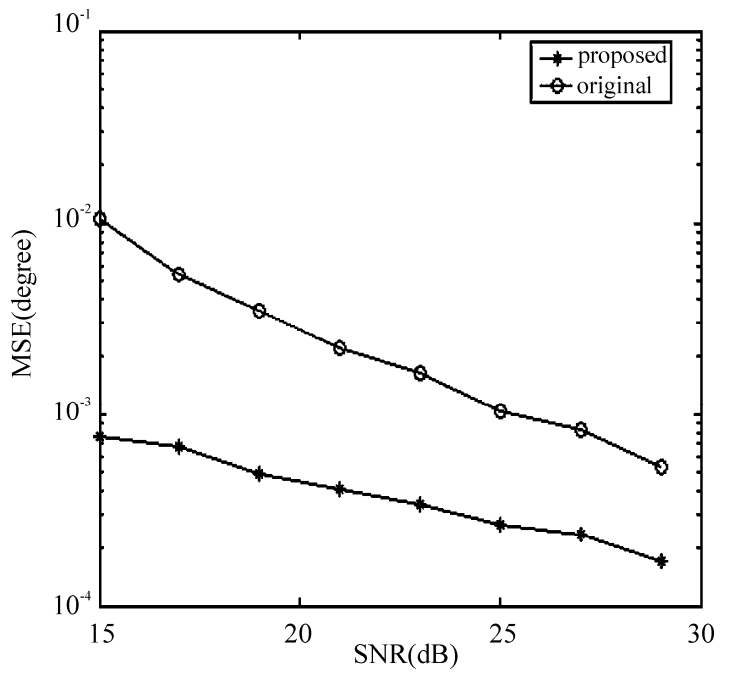

Figure 8. MSE of proposed and original method.

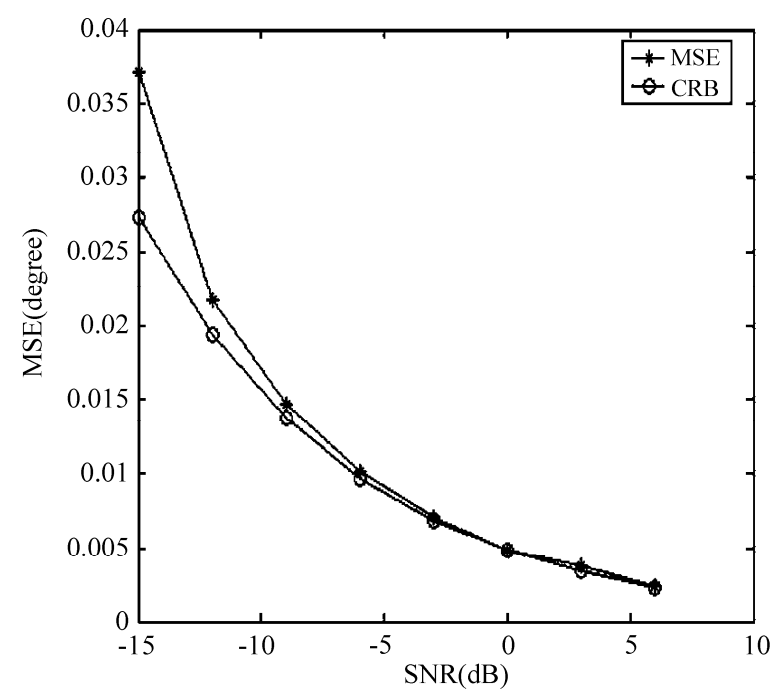

Figure 9. MSE and CRB of proposed method.

shown in Figure 8. Obviously, the accuracy of our method has certain improvement comparing with the method proposed in the Reference [4].

In same assumption, the input SNR various from $-15 \mathrm{~dB}$ to $6 \mathrm{~dB}$ with an interval $3 \mathrm{~dB}, 100$ times Monte-Carlo simulations are performed at each level of the SNR, MSE of the first signal and CRB are shown in Figure 9. It can be seen, the MSE of proposed method is closed to the CRB even at the lower SNR.

\subsection{2-D DOA Estimation Simulation}

\subsubsection{2-D Estimation RMSE Simulation}

$D=2$ two far-field LFM sources shoot the $N=20$ UCA with the angle information $\left\{\left(\theta_{1}=60^{\circ}, \beta_{1}=50^{\circ}\right)\right.$, $\left.\left(\theta_{1}=30^{\circ}, \beta_{1}=70^{\circ}\right)\right\}$. The signal model is: $\omega_{1}=$ 
$200 \pi \mathrm{Hz}, \quad \mu_{1}=300 \pi \mathrm{Hz} / \mathrm{s} ; \quad \omega_{2}=200 \pi \mathrm{Hz}, \quad \mu_{2}=$ $-900 \pi \mathrm{Hz} / \mathrm{s}$. Sampling rate is $f_{s}=900 \mathrm{~Hz}$, number of snapshots is 1024 , and the cover filter length is $2 L=300$. The Figure 10(a) gives the 2-D DOA estimation of signal one in the OdB SNR.

Change the input SNR range from $0 \mathrm{~dB}$ to $20 \mathrm{~dB}$ with the interval $5 \mathrm{~dB}$, firstly perform big step search to obtain the rough DOA estimation. Then run the high differentiation search with the 0.001 rad step. Run 300 time Monter-Carlo experiment respectively, the RMSE (root mean square error, RMSE) comparison curves of the proposed algorithm and literature one can be seen in Figure 10(b). The accuracy of our method has certain improvement compared to the original algorithm.

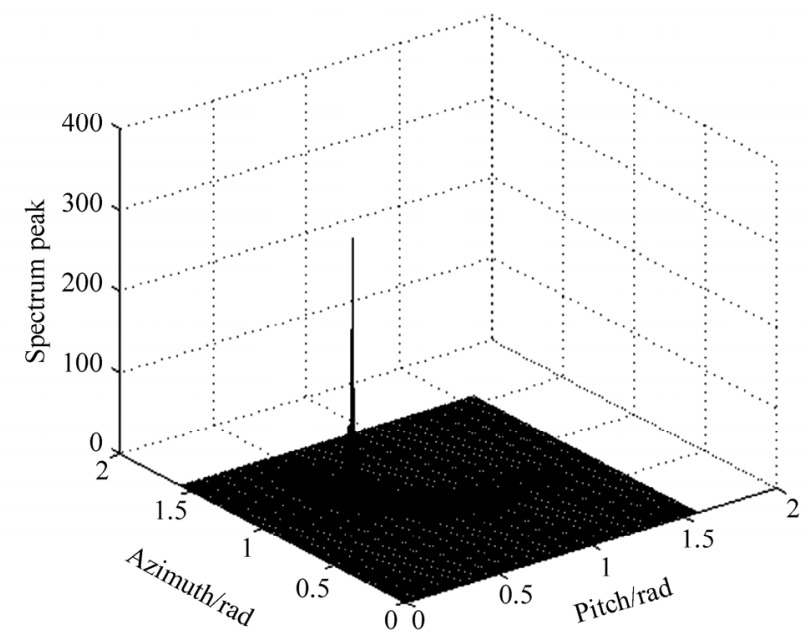

(a)

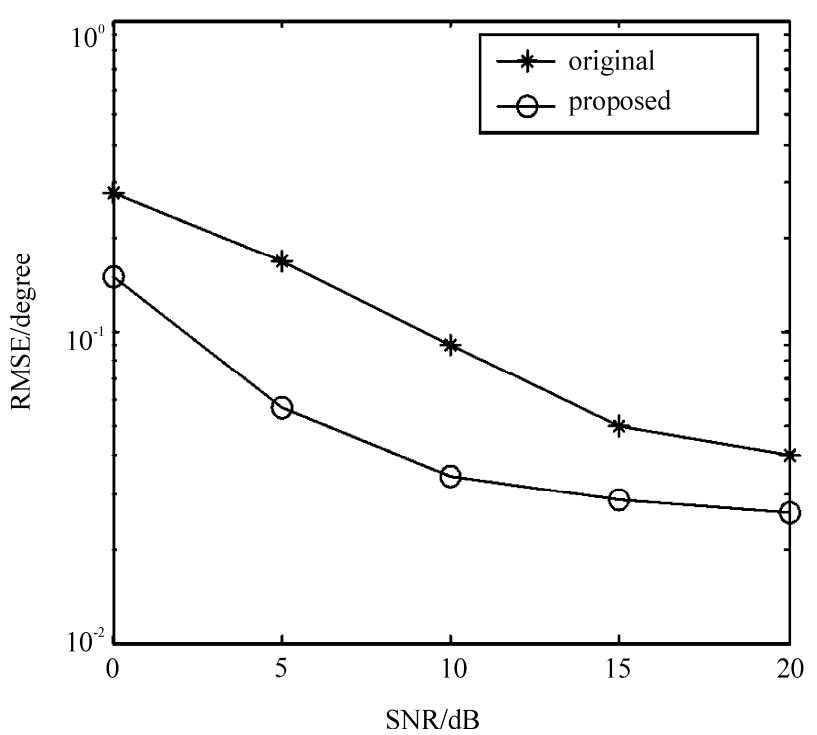

(b)

Figure 10. (a) 2-D DOA estimation using UCA, (b) RMSE comparison curves using UCA.

\subsubsection{2-D Estimation Performances in Complex Channel and Simulation}

In mobile communication system, the proposed algorithm is applied to the complex channel which the multipath and Doppler shift is existed simultaneously. In the same simulation conditions, viz. the random signal source model is:

$$
s_{k}(t)=\sum_{e=1}^{E} M_{e} \exp \left(j f_{e} t\right) \exp \left[j\left(\omega_{k}\left(t-\delta_{e}\right)+\mu_{k}\left(t-\delta_{e}\right)^{2} / 2\right)\right]
$$

where, $M_{1}=1, M_{2}=0.9$, Doppler frequency shift is $f_{1}=0, f_{2}=2$, multi-path delay is $\delta_{1}=0, \delta_{2}=1 / 900$.

When the SNR is $0 \mathrm{~dB}$, the simulation result of signal one in most powerful path can be shown in Figure 11(a).

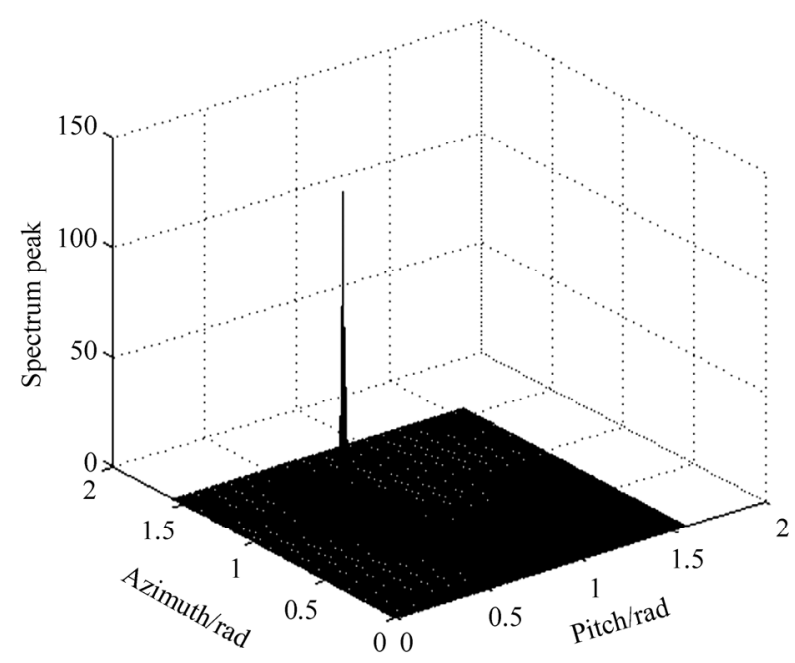

(a)

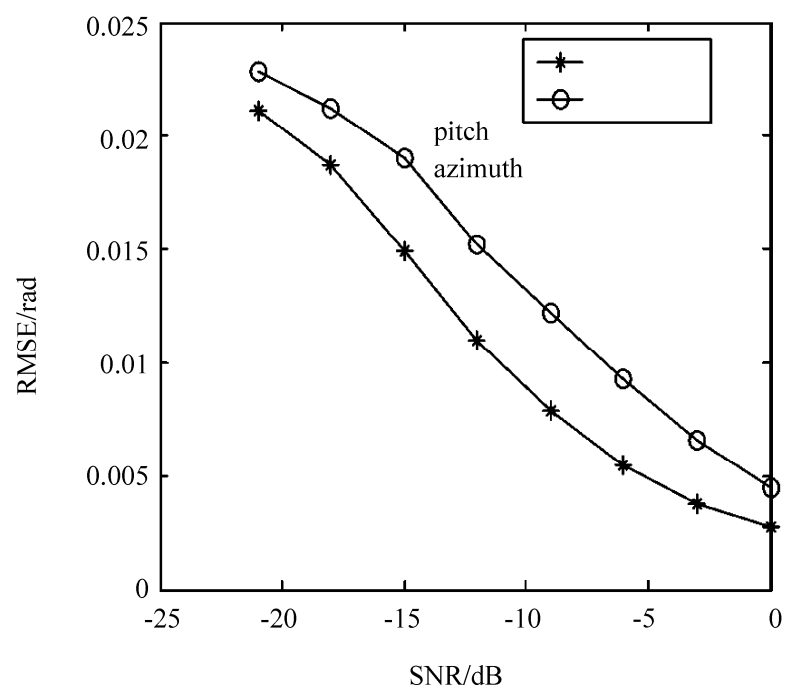

(b)

Figure 11. (a) 2-D DOA estimation in complex channel, (b) RMSE curves in complex channel. 
Change the input SNR range from $-21 \mathrm{~dB}$ to $0 \mathrm{~dB}$ with the interval $3 \mathrm{~dB}$. Run 300 time Monter-Carlo experiment respectively, the RMSE comparison curves of signal one can be seen in Figure 11(b), which can show that the proposed algorithm is also effective in complex channel.

\section{Conclusions}

Analyzing the definition and characteristics of the FRFT, a novel DOA estimation algorithm has been presented. In the implementation of the method, mask operation is introduced to simply the filtering procedure with no accuracy degradation. Demodulation operation is used to extend the application range of the traditional estimate method without performance loss. Compared with other methods, the veracity has certain improvement while the cross-terms and interpolation are avoided. The proposed is also expanded to the 2-D DOA estimation using UCA, which is suitable for the multi-path and Doppler frequency shift complex environment. In addition, the proposed the method can be also applied to DOA estimation of LFM signals in colored noise or near-field environment, which is not described in this paper.

The theoretical analyses about the error and CRB are also provided and verified by simulation results thus making this method more reliable in theory and in practice, meanwhile enrich the principle and application of the FRFT. As for the optimization, the 2-D Cramer-Rao Bound of the proposed algorithm is the further research direction.

\section{Acknowledgements}

The authors would like to thank the reviewers for their detailed comments on earlier versions of this paper.

\section{References}

[1] G. Wang and X. G. Xia, "Iterative algorithm for direction of arrival estimation with wideband chirp signals," IEE Proceedings of Radar, Sonar, Navig, Vol. 147, No. 5, pp. 233-238, 2000.

[2] A. Belouchirani and M. G. Amin, "Time-frequency MUSIC [J]," IEEE Signal Processing Letters, Vol. 6, No. 5, pp. 109-110, 1999.
[3] A. B. Gershman and M. G. Amin, "Wideband direction of multiple chirp signals using spatial time-frequency distributions," IEEE Signal Processing Letters, Vol. 7, pp. 152-155, June 2000.

[4] R. Tao and Y. S. Zhou, "A novel method for the DOA estimation of wideband LFM sources based on FRFT," Transactions of Beijing Institute of Technology, Vol. 25, No. 10, pp. 895-899, 2005.

[5] P. Stoica and A. Nehorai, "Music, maximum likelihood, and cramer-rao bound," IEEE Transactions on ASSP, Vol. 37, No. 5, May 1989.

[6] V. Namias, "The fractional Fourier transform and its application in quantum mechanics [J]," IMA Journal of Applied Mathematics, No. 25, pp. 241-265, 1980.

[7] L. B. Almeida, "Fractional Fourier transform and time-frequency representations [J]," IEEE Transactions on Signal Processing, Vol. 42, No. 11, pp. 3084-3091, 1994.

[8] Y. Q. Dong, R. Tao, S. Y. Zhou, et al., "SAR moving target detection and imaging based on fractional Fourier transform," Acta Armamentarii (in Chinese), Vol. 20, No. 2, pp. 132-136, 1999.

[9] L. Qi, R. Tao, S. Y. Zhou, et al., "Adaptive time-varying filter for linear FM signal in fractional Fourier domain," Proceedings of the 6th ICSP, Posts and Telecommunications Press, Beijing, pp. 1425-1428, 2002.

[10] R. Tao, L. Qi, and Y. Wang, "Principle and application of the fractional Fourier transform," Tsinghua Publishing Company, Beijing, 2004.

[11] H. M. Ozaktas, O. Arikan, M. A. Kutay, et al., "Digital computation of the fractional Fourier transform," IEEE Transactions on Signal Processing, Vol. 44, No. 9, pp. 2141-2150, 1996.

[12] L. Qi, R. Tao, S. Y. Zhou, et al., "Detection and parameter estimation of multicomponent LFM signal based on the fraction Fourier transform [J]," Science in China (Series E), Vol. 47, No. 2, pp. 184-198, 2004.

[13] X. D. Zhang, et al., Modern Signal Processing (Second editor), Tsinghua Publishing Company, Beijing, 2002.

[14] C. P. Mathews, "Eigenstructure techniques for 2-D angle estimation with uniform circular arrays," IEEE Transactions on Signal Processing, Vol. 42, No. 9, pp. 23952407, September 1994.

[15] L. M. Yang "DOA estimation for wideband sources based on UCA," Journal of Electronics, China, Vol. 23, No. 1, January 2006. 